\title{
Quantization of a free particle interacting linearly with a harmonic oscillator
}

\author{
Thomas Mainiero \\ Department of Physics, California Institute of Technology, Pasadena, California 91125, USA \\ Mason A. Porter \\ Oxford Centre for Industrial and Applied Mathematics, Mathematical Institute, University of Oxford, \\ Oxford OX1 3LB, United Kingdom
}

(Received 13 February 2007; accepted 1 November 2007; published online 28 December 2007)

\begin{abstract}
We investigate the quantization of a free particle coupled linearly to a harmonic oscillator. This system, whose classical counterpart has clearly separated regular and chaotic regions, provides an ideal framework for studying the quantization of mixed systems. We identify key signatures of the classically chaotic and regular portions in the quantum system by constructing Husimi distributions and investigating avoided level crossings of eigenvalues as functions of the strength and range of the interaction between the system's two components. We show, in particular, that the Husimi structure becomes mixed and delocalized as the classical dynamics becomes more chaotic. (C) 2007 American Institute of Physics. [DOI: 10.1063/1.2819060]
\end{abstract}

Typical classical Hamiltonians systems are neither fully integrable nor fully chaotic, but instead possess mixed dynamics, with islands of stability situated in a chaotic sea. In this paper, we investigate the quantization of a recently-studied system with mixed dynamics. ${ }^{1}$ This example consists of a free particle that moves around a ring that is divided into two regions. At the boundaries between these regions, the particle is kicked impulsively by a harmonic oscillator (in a manner that conserves the system's total energy), but the particle and oscillator otherwise evolve freely. Although the system is not generic, its separation into regular and chaotic components also allows more precise investigations (both classically and quantum-mechanically) than is typically possible, making this an ideal example to achieve a better understanding of the quantization of mixed systems. By examining avoided level crossings and Husimi distributions in the quantum system, we investigate the quantum signatures of mixed dynamics, demonstrating that the Husimi structures of nearby states become mixed and delocalized as chaos becomes a more prominent feature in the classical phase space.

\section{INTRODUCTION}

Investigations of the quantization of chaotic systems have become increasingly prevalent as physicists conduct more experiments at small scales and design an increasing number of devices that exploit the physics at such scales. ${ }^{2-6}$ Experiments on quantum chaos, conducted using microwave cavities, ${ }^{7,8}$ atom optics, ${ }^{9,10}$ and other systems, have examined phenomena that are both fundamental and diverse-ranging from the decay of quantum correlations ${ }^{9}$ to localization in quantum wave functions ${ }^{7}$ and chaotic scattering. ${ }^{4}$ Despite this wealth of research, however, it is still not entirely clear how to understand the notion of chaos in quantum mechanics. Sensitive dependence on initial conditions and the exponentially fast divergence of nearby trajectories-key components for defining classical chaos-cannot be used to define quantum chaos. ${ }^{2}$ Nevertheless, quantum analogs of classically chaotic systems do possess identifying features, so the quantizations of chaotic systems can be distinguished from the quantizations of integrable (regular) ones.

Typical classical Hamiltonian systems are neither fully chaotic nor fully regular; rather, they have "mixed" dynamics (i.e., a divided phase space), with islands of stability [Kolmogorov-Arnold-Moser (KAM) islands] situated in a chaotic sea. Because generic mixed systems are very difficult to analyze, there have been numerous attempts to construct Hamiltonian systems with mixed dynamics that allow an exact, rigorous analysis. Previously studied examples include billiards, ${ }^{11-15}$ Fermi accelerators and bouncing-ball models, ${ }^{16-18}$ and kicked rotors and tops. ${ }^{3,19-21}$

In the present paper, we investigate the quantization of a one-dimensional free particle interacting linearly with a onedimensional harmonic oscillator. This recently studied example has mixed dynamics with well-separated integrable and chaotic regions. ${ }^{1}$ The clean separation between different types of behavior helps simplify comparisons between the dynamics of the classical system and that of its quantization and makes this system a very illuminating one for studying the quantization of systems with mixed dynamics. The investigation of classical-quantum correspondences is extremely difficult for generic mixed systems, which possess an infinite hierarchy of KAM islands and intricately mixed chaotic and integrable regions. This makes identifying features of chaos in carefully chosen examples particularly important.

The rest of this paper is organized as follows. First, we briefly review the classical system studied in Ref. 1. We then quantize this system and examine its sharp and broad avoided level crossings as the relative length of the interaction versus noninteraction region is varied. We illustrate our observations using Husimi distributions, which also allow a comparison with the classical dynamics. Finally, we summarize our results and present additional technical details of our investigation in two appendices. 


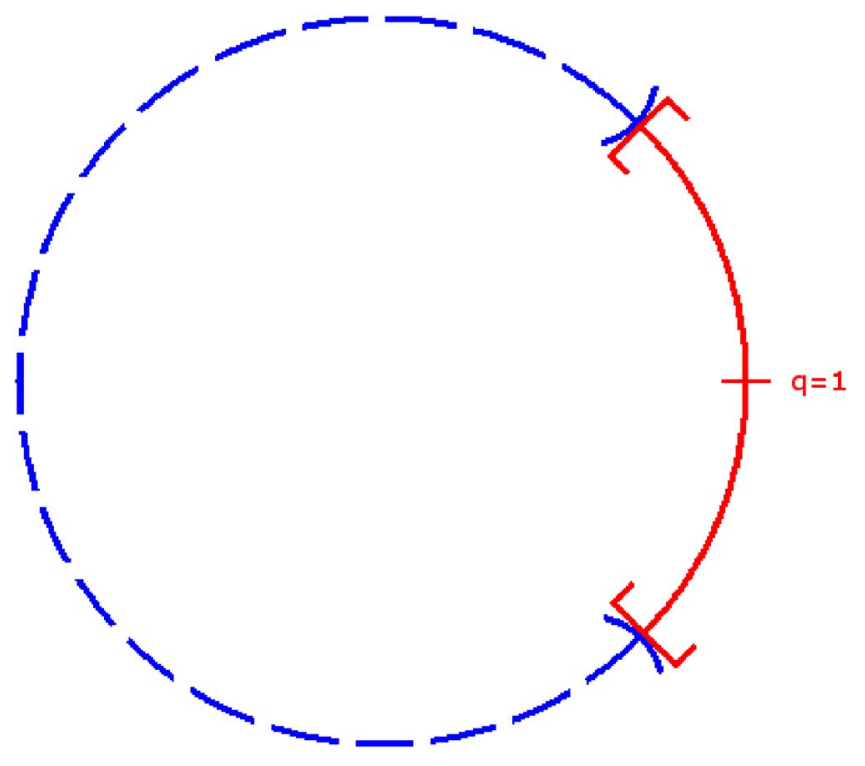

- - Uncoupled Region (Length L)

- Interaction Region (Length 2)

FIG. 1. (Color online) The configuration space of the particle. Its position on the ring is denoted $q$.

\section{THE CLASSICAL SYSTEM}

Motivated by investigations of electron-phonon interactions in condensed matter physics, ${ }^{22-30}$ De Bièvre, Parris, and Silvius recently performed an analysis of a closed (classical) Hamiltonian system consisting of an interacting onedimensional free particle and a one-dimensional harmonic oscillator. $^{1}$ The example they investigated consists of a classical particle moving on a ring divided into two sections (see Fig. 1): a region of length 2 called the "interaction region" and one of length $L$ called the "noninteraction" (or "uncoupled") region. At the boundaries between these regions, the particle is kicked impulsively by a harmonic oscillator (which moves on a line rather than on the ring), but the system behaves as if the particle and oscillator are otherwise uncoupled. However, the harmonic oscillator is centered at different equilibrium points in the two regions; as discussed below, the equilibrium position in the interaction region depends on the strength of the interaction.

The (nondimensionalized) Hamiltonian describing this system is

$$
H=\frac{1}{2}\left(p^{2}+\Pi^{2}+\Phi^{2}\right)-\alpha \Phi \chi(q),
$$

where $p$ and $\Pi$ are, respectively, the particle and oscillator momenta; $q$ and $\Phi$ are, respectively, the particle and oscillator positions; $\alpha$ describes the strength of the particleoscillator interaction; and $\chi(q)$ takes the value 1 in the interaction region and 0 everywhere else. We choose coordinates so that the interaction and noninteraction regions occur, respectively, when $q \in[0,2]$ and $q \in(2,2+L)$, where $L$ is the length of the noninteraction region and $2+L$ is identified with 0 . The only system parameters that can be varied are $\alpha$ and $L$.
Let us review some of the basic qualitative dynamics of Eq. (1). ${ }^{1}$ The system achieves its ground state when the particle is in the interaction region and the oscillator and particle are both at rest. When transitioning between the interaction and noninteraction regions, the particle's momentum changes discontinuously and its position changes continuously. When the particle enters the interaction region, the harmonic oscillator experiences an interaction force that shifts its equilibrium position from 0 to $\alpha$. (The oscillator's momentum and amplitude depend continuously on time.) One uses conservation of energy to compute the impulse that the oscillator imparts to the particle at the transition points. See Ref. 1 for further details.

The uncoupled Hamiltonian, given by Eq. (1) with $\alpha$ $=0$, is integrable. It possesses two independent integrals that correspond, respectively, to the energy of the harmonic oscillator and the momentum of the particle. The latter integral leads to an $S O(2)$ rotational symmetry in the particle's configuration space. Indeed, Eq. (1) is invariant under symplectic transformations

$$
T_{A}:(p, q, \Pi, \Phi) \mapsto(\operatorname{det}(A) p, A q, \Pi, \Phi), \quad A \in O(2),
$$

forming a symmetry group isomorphic to $O(2)$. However, for $\alpha \neq 0$ (i.e., the generic case), the Hamiltonian (1) no longer possesses the two integrals and is only invariant under the subset of the symplectic transformations [Eq. (2)] with either $A=1$ or $A$ a reflection about the line passing through $q=1$ and $q=1+L / 2$ (see Fig. 1). Such transformations form a subgroup of $O(2)$ isomorphic to $\mathbb{Z}_{2}=\{1,-1\}$, reducing this symmetry of the system to a parity symmetry about an axis of the ring in Fig. 1. Equation (1) also possesses a time-reversal symmetry, as it is invariant with respect to the transformation $(p, \Pi) \rightarrow(-p,-\Pi)$.

A particularly interesting facet of this system is the clean separation of the integrable and chaotic regions in its phase space. Phase portraits of the system possess two characteristic integrable regions (among other structures). The first, which exists for system energies varying from the groundstate energy to a critical positive energy, arises when the particle never leaves the interaction area. For small positive energies outside this integrable region, the motion appears to be fully chaotic without any additional KAM structures near the boundary between the two regions. ${ }^{1}$ The second integrable region is an elliptic KAM island centered on the point in the Poincare section corresponding to the orbit in which the particle traverses each section of the ring exactly once per period. More generally, the distinction between chaotic and integrable dynamics in this system is clear in a variety of situations.

\section{THE QUANTUM SYSTEM}

We quantize Eq. (1) using canonical quantization. ${ }^{31}$ In so doing, we assume that the particle and harmonic oscillator act as bosons with no internal degrees of freedom (DOF) and impose the following commutation relations: 


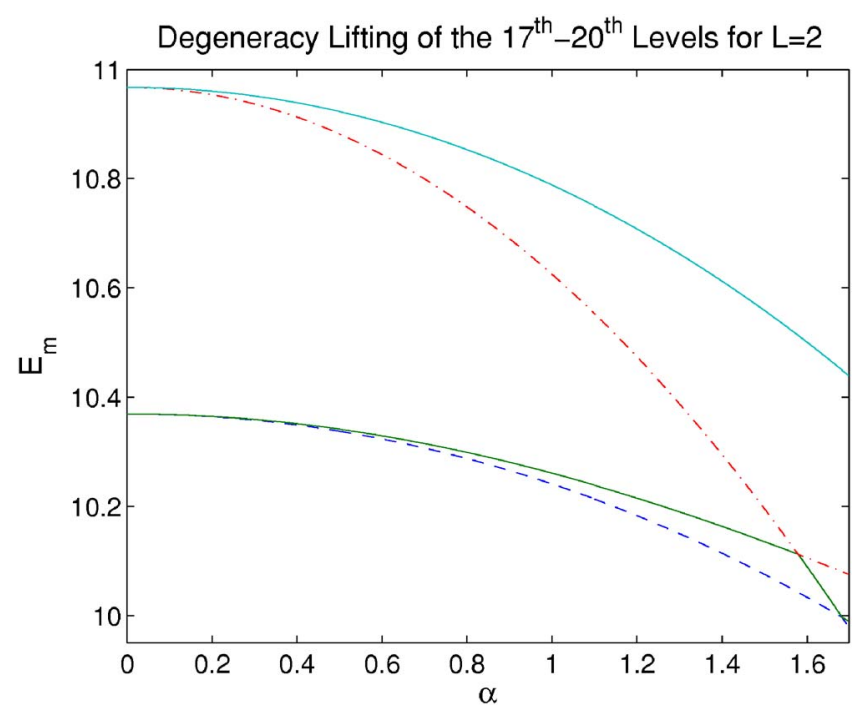

FIG. 2. (Color online) Lifting of the double degeneracy in the eigenenergies for $\alpha>0$.

$$
\begin{array}{ll}
{[p, q]=-i,} & {[p, \Pi]=0, \quad[\Pi, \Phi]=-i,} \\
{[q, \Phi]=0,} & {[p, \Phi]=0, \quad[q, \Pi]=0 .}
\end{array}
$$

With the coordinate-space identifications

$$
p=-i \frac{\partial}{\partial q}, \quad \Pi=-i \frac{\partial}{\partial \Phi},
$$

we obtain the quantum Hamiltonian

$$
H=\frac{1}{2}\left(-\frac{\partial^{2}}{\partial q^{2}}-\frac{\partial^{2}}{\partial \Phi^{2}}+\Phi^{2}\right)-\alpha \Phi \chi(q) .
$$

For the uncoupled system [Eq. (5), with $\alpha=0$ ], the timeindependent Schrödinger equation $H|\psi\rangle=E|\psi\rangle$ is separable, so one just needs to determine the eigenstates of the onedimensional harmonic oscillator (moving on a line) and the free particle confined to a ring of length $2+L$ as separate problems (both of which admit closed-form solutions). Let $\left\{\left|\psi_{n}^{\text {part }}\right\rangle\right\}_{n}$ and $\left\{\left|\psi_{k}^{\text {osc }}\right\rangle\right\}_{k}$ denote eigenstates of the particle and the harmonic oscillator, respectively, so that $\left\{\left|\psi_{n}^{\text {part }}\right\rangle \otimes\left|\psi_{k}^{\text {osc }}\right\rangle\right\}_{n, k}$ are eigenstates for the uncoupled system. In fact, these states form a basis for the Hilbert space of either the coupled or uncoupled system. We represent the Hamiltonian (5) as an infinite matrix using this basis (see Appendix A) and approximate its eigenvalues and eigenstates using those of a truncation of the matrix. Because the eigenenergies of the particle are doubly degenerate, the eigenenergies of the full uncoupled system are also doubly degenerate. Second-order perturbations in $\alpha$ lift this degeneracy for $\alpha>0$ and $L \neq 2$; numerical calculations indicate that this degeneracy is also lifted for $L=2$ (see Fig. 2).

The uncoupled quantum Hamiltonian commutes with both the particle momentum $p$ and the Hamiltonian $H_{\text {osc }}$ $=\left(\Pi^{2}+\Phi^{2}\right) / 2$ describing an isolated harmonic oscillator; these are quantized versions of the two independent integrals of the classical Hamiltonian. The uncoupled quantum Hamiltonian is thus integrable. ${ }^{32,33}$ The symmetry-breaking of the classical Hamiltonian is mirrored in the quantum system, as $[H, p]=\alpha \Phi[\chi(q), p] \neq 0$ and $\left[H, H_{\text {osc }}\right]=\alpha \chi(q)\left[\Pi^{2}, \Phi\right] / 2 \neq 0$ for $\alpha>0$ and generic values of $L$. The parity symmetry in $q$ and the time-reversal symmetry, described for the coupled classical Hamiltonian, are still present in Eq. (5), but there are no obvious continuous symmetries for $\alpha>0$.

\section{AVOIDED CROSSINGS}

As the coupling parameter $\alpha$ is varied, the eigenvalues of Eq. (5) can approach each other very closely or even cross. If the Hamiltonian is invariant under a symmetry transformation for a certain range of $\alpha$, it can be block-diagonalized by exploiting this symmetry. One does this by choosing each block to be invariant under the symmetry transformation. Energy levels belonging to different blocks can cross as $\alpha$ is varied. ${ }^{3,434}$ On the other hand, if a quantum Hamiltonian has no symmetries other than time reversal, then such a level crossing is called an "accidental degeneracy" and requires the confluence of two parameters. ${ }^{5}$ In this case, most levels that approach each other end up avoiding one another instead of crossing.

Classical chaotic systems have fewer constants of motion than DOF and thus have fewer symmetries than integrable systems with the same number of DOF. One expects the quantization of these two situations to exhibit signatures of this difference. ${ }^{32,33}$ Hence, the quantization of a chaotic system should possess fewer level crossings than the quantization of an integrable one, and the presence of numerous avoided crossings between energy levels provides a signature of chaotic regions in the classical system. Indeed, avoided level crossings are typical features of quantum chaotic systems. ${ }^{35-39}$

Figure 3(a) shows the first 100 energy levels as a function of $\alpha$ in a system with $L=2$ using an $\alpha$-step size of 1.5 $\times 10^{-3}$ and a $2916 \times 2916$ truncated Hamiltonian matrix. (The $\alpha$-step size is the distance between successive values of $\alpha$ for which we calculate eigenvalues and eigenvectors.) One observes a multitude of apparent level crossings. Refining the numerical computation at some of these apparent crossings shows that they are actually avoided crossings at which the slopes of the energy-level curves are exchanged. Avoided crossings of this nature are known as "sharp" avoided crossings. ${ }^{5,38}$ In passing through such avoided crossings, the participating levels exchange their eigenstate structures, behaving as though they had entered a level crossing. ${ }^{34}$ As we discuss in more detail later, we have verified numerically that this indeed occurs for our system. Similar phenomena have also been observed in other systems, such as a sinusoidally driven particle in a square potential well ${ }^{38}$ and a hydrogen atom in a strong magnetic field. ${ }^{35}$

Figure 3(b) shows a magnification of an avoided crossing between the 18th and 19th levels from Fig. 3(a) using a refined $\alpha$-step size of $2 \times 10^{-6}$. In general, the maximum $\alpha$-step size at which the avoided crossings in Fig. 3(a) can be resolved is $O\left(10^{-6}\right)$. As a result, it is time-consuming to verify numerically that all of the apparent crossings are actually very sharp avoided crossings. Although we have only observed avoided crossings, we have not ruled out that actual level crossings might occur. Using parity symmetry, the Hamiltonian (5) can be separated into the direct sum of two 


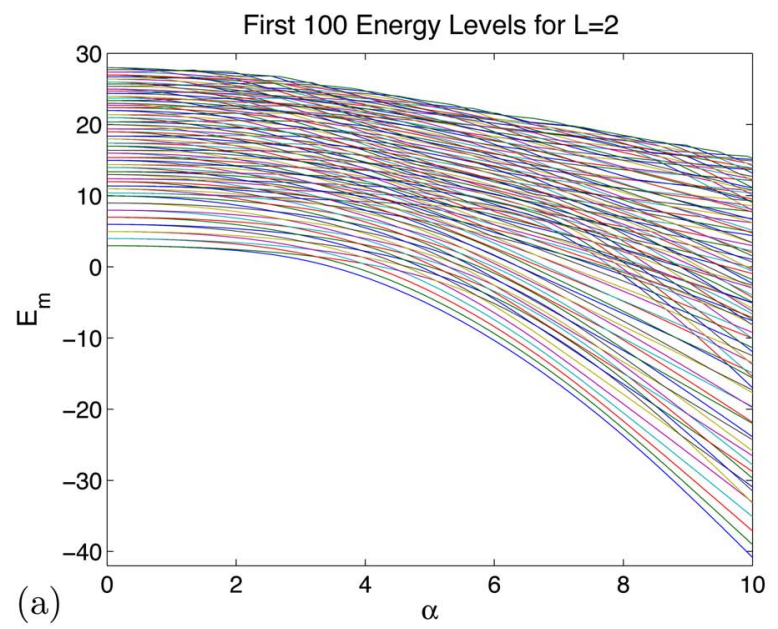

Avoided Crossing Between the $18^{\text {th }}$ and $19^{\text {th }}$ Levels for $L=2$

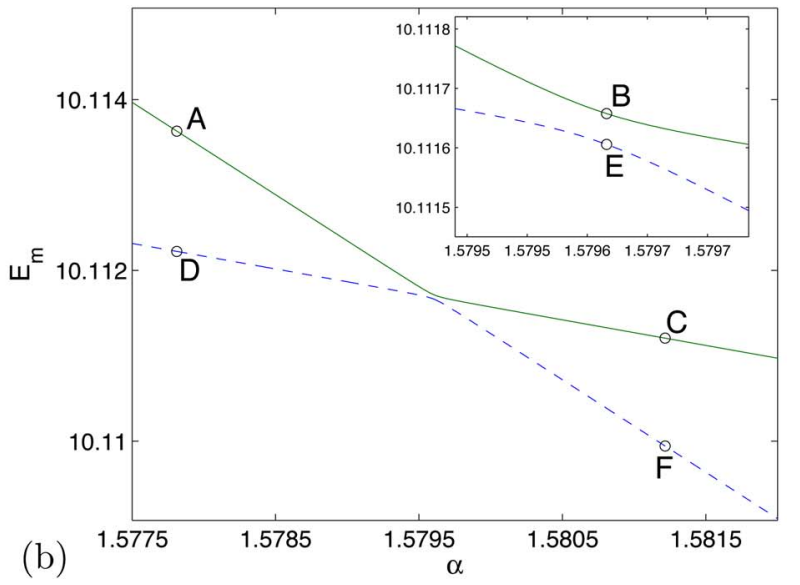

FIG. 3. (Color online) (a) The first 100 energy levels as a function of the interaction strength $\alpha$ for Eq. (5) with a noninteraction region of length $L$ =2. (b) Magnification of the avoided crossing between the 18th and 19th levels from panel (a). The inset shows a further magnification, and the labels designate where we calculated Husimi distributions (see Fig. 9).

blocks. As $\alpha$ is varied for a fixed value of $L$, energy levels from different blocks might cross.

Figure 4(a) shows the first 100 energy-level curves for $L=12$. Our numerical computations, for which we used a $4096 \times 4096$ truncated Hamiltonian matrix, verify that the frequency of avoided crossings increases as $L$ grows. Even more interesting are the broad avoided crossings, which become more prevalent as $\alpha$ increases. Figure 4(b) shows energy-level curves for $\alpha \in[5.8,7.2]$. The $\alpha$-step size for which the sharp avoided crossings in this figure can be resolved is $O\left(10^{-3}\right)$, about 1000 times larger than that required to resolve the sharp avoided crossings in Fig. 3(a). We have also observed that the prevalence of broad avoided crossings increases as $L$ increases. In general, broad avoided crossings tend to occur in nearly isolated clusters in which only a subset of the initial slopes of the participating levels are exchanged after the sequence of crossings in the cluster. ${ }^{40}$ This behavior is caused by the presence of "overlapping" avoided crossings in the cluster. (By "overlapping," we mean that there are ranges of $\alpha$ values for which multiple avoided crossings in the cluster are taking place.) Such clusters usu-
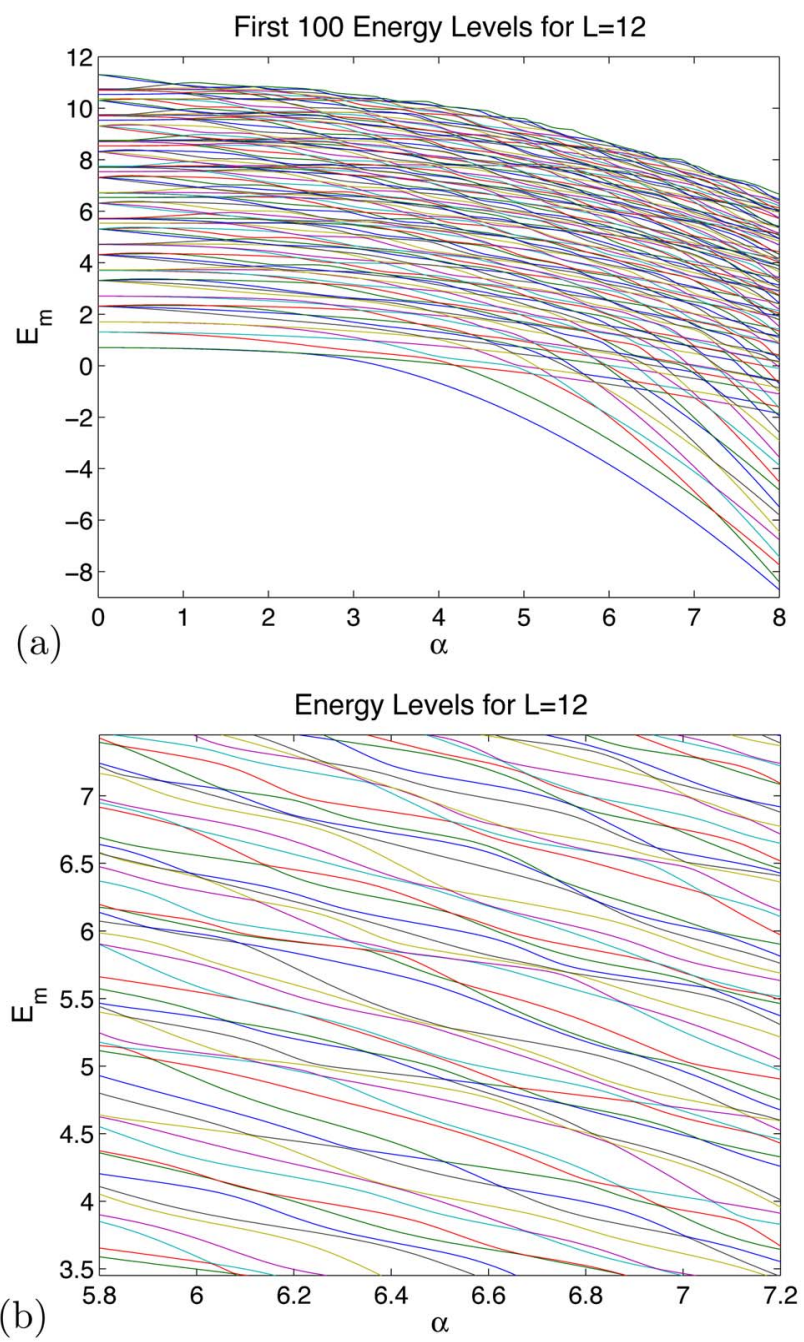

FIG. 4. (Color online) (a) The first 100 energy levels as a function of $\alpha$ for Eq. (5) with a noninteraction region of length $L=12$. (b) Magnification of panel (a) illustrating broad avoided crossings.

ally induce nontrivial structural exchanges between the participating eigenstates and are suspected to be an indication of chaos. ${ }^{41,42}$ Indeed, prior work on a sinusoidally driven particle in a square potential well ${ }^{38}$ indicates that broad avoided crossings produce superpositions of eigenstate structure rather than complete exchanges. We will use the term "mixing" to refer to such superpositions.

An example of an isolated cluster of avoided crossings is shown in Fig. 5. This cluster occurs for $L=12$ and shows the 119th-121st levels as they experience two sharp avoided crossings and one broad one. A broad avoided crossing begins between the 120th and 121st levels, but as $\alpha$ is increased, two sharp avoided crossings cause structural exchanges that induce a broad avoided crossing between the 119th and 120th levels. The figure gives the appearance of a broad avoided crossing overlapping with the second sharp avoided crossing. Consequently, the slopes of the participating levels in the second sharp avoided crossing are not completely exchanged. We have observed that such behavior often arises in avoided crossings (one of which is almost always broad) that occur simultaneously as $\alpha$ is varied. 
Cluster of Avoided Crossings between the $119^{\text {th }}-121^{\text {st }}$ Energy Levels for $L=12$

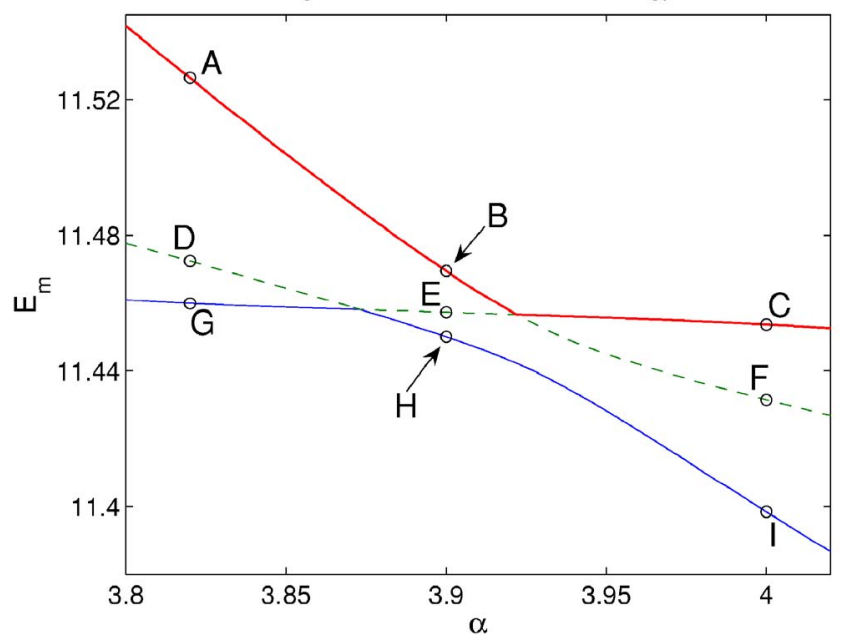

FIG. 5. (Color online) An isolated cluster of two sharp avoided crossings and a broad avoided crossing between the 119th, 120th, and 121st energy levels for $L=12$. The incoming slope of the 119th level is imparted to the outgoing slope of the 121st. The labels designate locations at which we calculated Husimi distributions (see Fig. 10).

While the complex interactions between energy levels (as a function of $\alpha$ ) may make it impossible to precisely identify broad versus sharp avoided crossings in isolated clusters, approximate distinctions such as that just discussed allow one to classify these interactions and determine their role in structural exchanges between eigenstates (see the discussion below).

One can gain insight into the energy-level dynamics for highly excited states (i.e., in the deep semiclassical regime) using level-spacing statistics. ${ }^{2,43}$ Once desymmetrized, completely integrable systems obey Poisson statistics and completely chaotic ones obey Wigner statistics. The quantization of mixed systems with well-separated integrable and chaotic regions (such as the present one) have energy-level statistics that interpolate between these two extremes, with a distribution of a precise form that is conjectured to follow BerryRobnik statistics. ${ }^{44-46}$ In the present paper, we focus on eigenstate structures rather than semiclassical dynamics, and we accordingly investigate Husimi distributions below and leave the analysis of energy-level statistics for a future publication.

\section{HUSIMI DISTRIBUTIONS}

Although there is no equivalent of classical phase space trajectories in quantum mechanics, there are suitable analogs. In particular, the Husimi distribution is often used in the study of quantum chaos. ${ }^{2,47}$ Given a quantum state $|\psi\rangle$, its Husimi distribution $H_{\psi}(p, q)$ is defined by the projection of $|\psi\rangle$ onto a coherent state $\left|\psi_{(p, q)}\right\rangle$ localized around $(p, q)$; that is, $H_{\psi}(p, q) \propto\left|\left\langle\psi_{(p, q)} \mid \psi\right\rangle\right|^{2}$. For a system with Euclidean topology, a coherent state localized at $(p, q)$ is a Gaussian with position-space representation localized around $q$ and momentum-space representation localized around $p$. The system (5) possesses a cylindrical phase-space topology, as the particle position $q$ is a periodic variable and the momentum $p \in \mathbb{R}$. In Appendix B, we construct the coherent state for this topology from the Euclidean coherent state. ${ }^{48}$

Coherent states provide excellent quantum analogs of classical particles when visualized as wave packets that minimize the position-momentum uncertainty product. The projection onto these particle-like states can thus be viewed intuitively as a sort of classical smearing. One then interprets the Husimi distribution as a probability distribution in phase space, ${ }^{49}$ allowing one to understand the dynamics of a quantum system in an analogous manner to phase portraits [in particular, Poincaré surfaces of section ${ }^{17,50}$ (SOS)] of its classical counterpart. See Refs. 49, 51-53 for additional discussions and applications. For the classical system (1), we plot surfaces of section for various values of $q$ on the projection of phase space onto the harmonic oscillator's coordinates [i.e., on $(\Phi, \Pi)$-space]. We often take the SOS at $q=1$, where we impose the further restriction $p>0$ due to the $p$-reversal symmetry of such sections that results from the parity and time-reversal symmetries. ${ }^{1}$ As discussed in Appendix B, we calculate Husimi distributions for eigenstates of the Hamiltonian (5) and project them onto the quantum oscillator phase space. We compare Husimi distributions to corresponding classical SOS using relevant eigenstate energies and the same values for the system parameters $\alpha$ and $L$.

\section{A. Localization around classical features}

To investigate the signatures of chaotic and integrable dynamics on the quantum system, we compare quantum $\mathrm{Hu}$ simi structures to corresponding classical SOS., ${ }^{2,38,49,53}$ As expected, eigenstates sometimes show localization around distinctive features of an SOS. A prominent manifestation that often occurs is a strong localization around KAM islands, which is sometimes accompanied with Husimi density throughout the chaotic sea. Figure 6 shows two such states: In panel (a), the Husimi structure is strongly localized around quasiperiodic orbits in an integrable region of the classical SOS; in panel (b), the structure is strongly localized in and around multiple KAM islands. In each figure, we display the Husimi distribution as a contour plot that varies from black (zero probability) to white (about $10^{-3}$ ), with lighter regions having higher probability than darker regions. We overlay the classical SOS in turquoise (online only). We observe that the quantum manifestation of a classical integrable region is a Husimi structure strongly localized inside the region. By analogy with the classical dynamics, we refer to such states as "regular."

Figure 7(a) shows an eigenstate whose Husimi structure is located in the chaotic sea of its corresponding classical SOS (particularly around the edge on the left half). We observe delocalization of the distribution throughout the chaotic sea, providing a signature of the predominantly chaotic dynamics of the classical SOS. By analogy with the classical dynamics, we refer to quantum states with such structures as "chaotic." For Husimi distributions taken at $q=1$, we have only observed structures localized around the left half of the available phase space. However, distributions constructed for $q$ values in the noninteraction region of phase space have structures with positive density throughout the available 

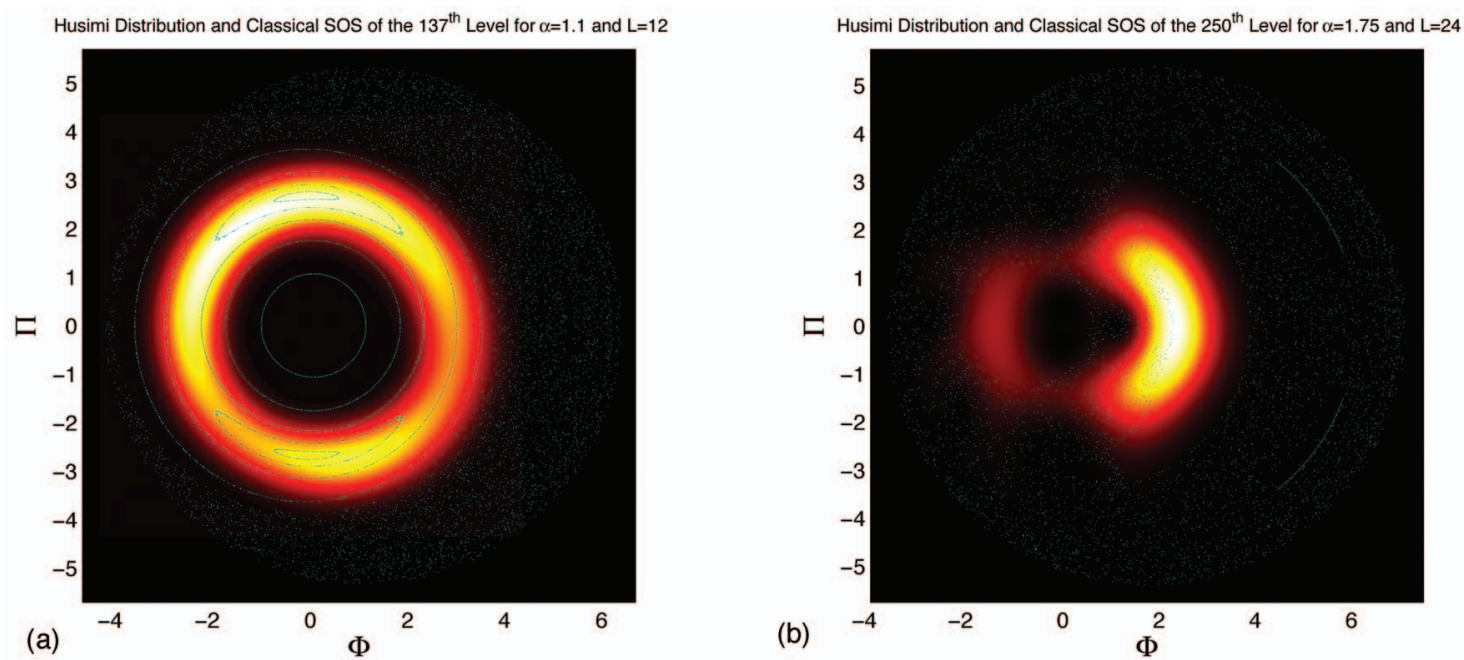

FIG. 6. (Color) (a) Husimi distribution localized around quasiperiodic orbits in a KAM island. Lighter regions have higher probabilities and black regions are ones with zero probability. The classical SOS is plotted in turquoise. To facilitate the comparison between the quantum and classical dynamics, we include plots of a few of the orbits in the integrable region. (b) Husimi distribution localized around multiple KAM islands.

phase space. Figures 7(b) and 8(a), for example, show Husimi distributions (and their corresponding classical SOS) for the chaotic state in Fig. 7(a) taken on the surfaces defined by $q=2+L / 4=12$ (one fourth of the way into the noninteraction region) and $q=2+L / 2=22$ (the middle of the noninteraction region), respectively. These Husimi distributions still display a strong localization on the left but nevertheless have a significant density throughout the available phase space.

The enhanced density around the left edge of the chaotic sea is present for all Husimi distributions with chaotic structures. We observe this as well for Husimi distributions taken in the noninteraction region. As illustrated in Fig. 8(b), this occurs even for large energies, for which the chaotic region in the left half of the SOS retreats to the edge of the available phase space. The location of this enhanced density coincides with the left boundary of the intersection between the available oscillator phase space in the interaction region and the

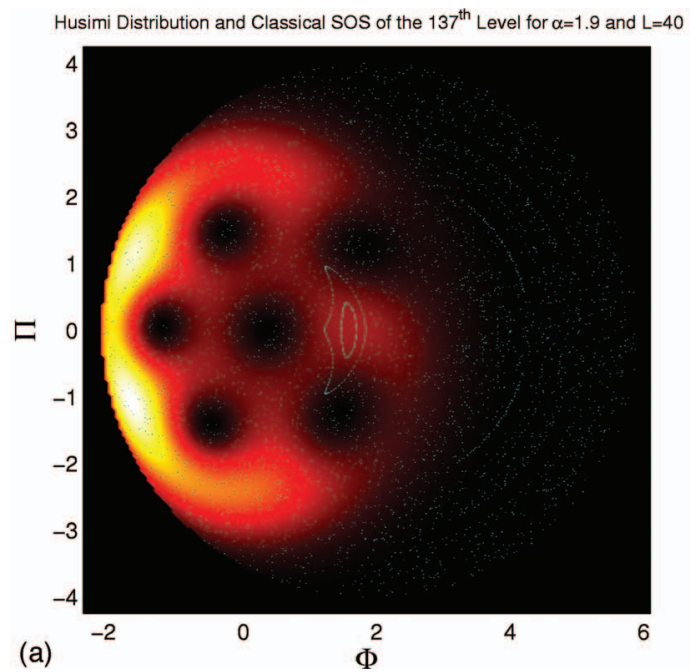

available oscillator phase space in the noninteraction region. As discussed in detail in Ref. 1, the portion of oscillator phase space corresponding to the interaction region is given by a disk-shaped region of radius $\sqrt{2 E+\alpha^{2}}$ centered at $(\alpha, 0)$ and that corresponding to the noninteraction region is given by a disk-shaped region of radius $\sqrt{2 E}$ centered at the origin. Comparing Figs. 7(a) and 7(b), we observe that this intersection in classical phase space occurs for $\Phi \approx-2$ between $\Pi$ $\approx-2$ and $\Pi \approx 2$. In both plots, the Husimi density is clearly enhanced in this area, in correspondence with the dynamics of the classical system.

In Fig. 8(b), we show a Husimi distribution that contains a mixture of regular and chaotic features. Although the regular and chaotic structures appear to be disconnected at first glance, one can see upon very close inspection that they are actually connected by small "bridges" with positive Husimi

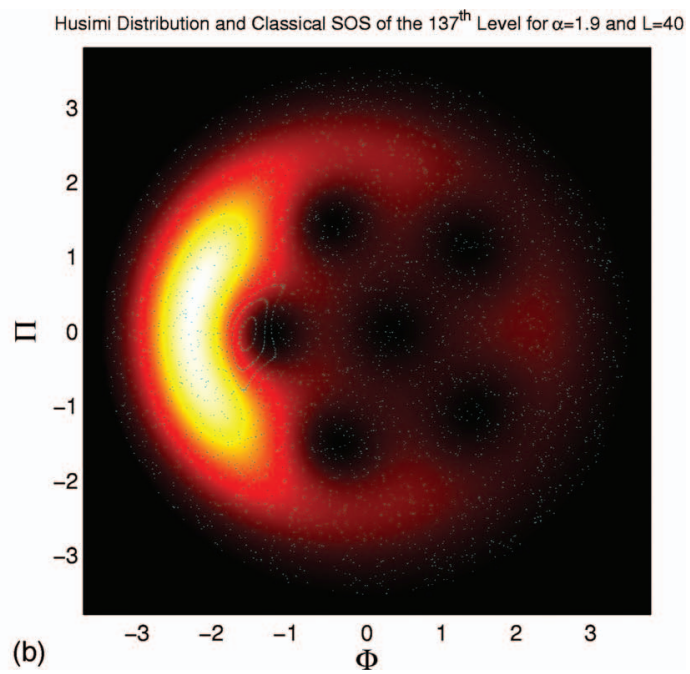

FIG. 7. (Color) (a) Husimi distribution delocalized throughout the chaotic sea on the left half of the plot. (b) Husimi distribution and classical SOS for the chaotic eigenstate in (a) taken on the surface defined by $q=2+L / 4=12$ (one fourth of the way into the noninteraction region). 

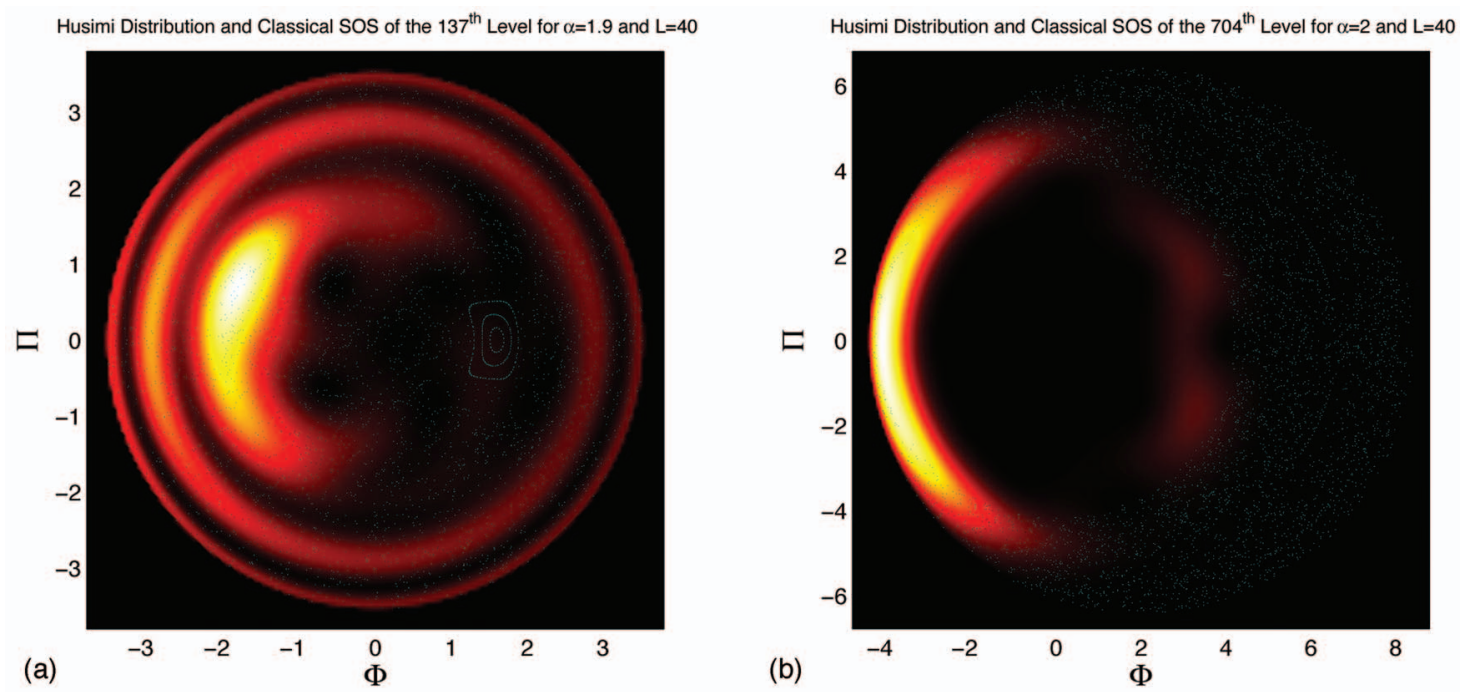

FIG. 8. (Color) (a) Husimi distribution and classical SOS for the chaotic eigenstate in Fig. 7 (a) taken on the surface defined by $q=2+L / 2=22$ (in the middle of the noninteraction region). (b) Husimi distribution showing both chaotic and integrable features while having only one connected component. The structure is relatively delocalized throughout the enclosed area on the left side of the available phase space.

density. Such bridges appear more prominently between the regular structures in Fig. 6(b). The bridges that are often observed between Husimi structures localized in different classical phase space regions (for example, between integrable and chaotic regions) serve as channels through which density continuously flows as $\alpha$ is varied through an avoided crossing. We did not observe any Husimi distributions in which the regular and chaotic components were completely disconnected.

In concluding this subsection, we remark that there is a considerable body of work on regular versus chaotic eigenfunctions. According to Percival's conjecture, eigenfunctions localize either on integrable or chaotic regions of the underlying classical phase space, so that the partial level density of regular states is given approximately by the fraction of volume of the integrable region relative to the available phase space. ${ }^{11,54,55}$ A recent paper by Marklof and O'Keefe ${ }^{56}$ contains rigorous results on such an extension of quantum ergodicity theory to a general class of quantum unitary maps whose underlying classical system has a divided phase space. It would be extremely interesting to obtain similar results for mixed systems like the present one.

\section{B. Exchange and mixing of Husimi structures at avoided crossings}

\section{Sharp avoided crossings}

In Fig. 9, we depict Husimi distributions for the 18th and 19th energy levels of Eq. (5) with $L=2$. This plot reveals the structural changes that occur as the two levels encounter the avoided crossing in Fig. 3(b). In the top panels, one can see that the 18th level is a regular state, whereas the 19th level is chaotic. The middle panels give snapshots near the closest point of the encounter. Here, the Husimi distributions appear

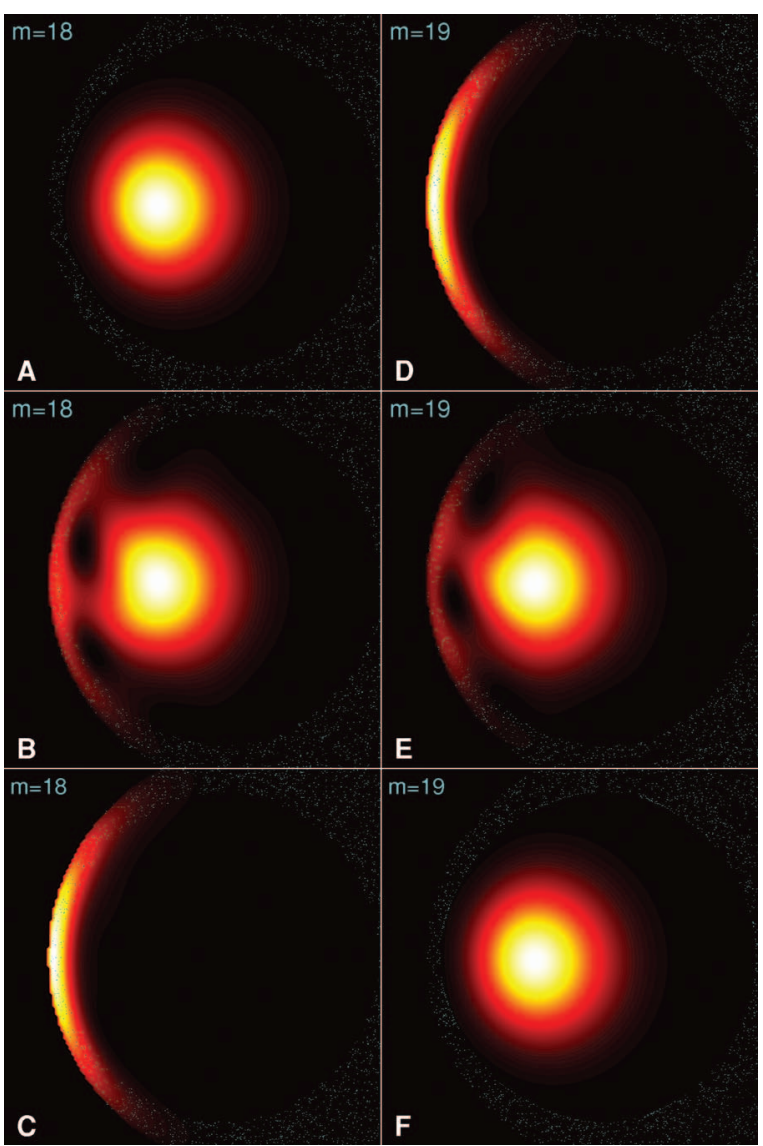

FIG. 9. (Color) Husimi structure exchange through the sharp avoided crossing shown in Fig. 3(b). The left and right columns show the Husimi distributions of the 18th and 19th levels, respectively. The harmonic oscillator momentum $\Pi$ is on the vertical axis and the oscillator position $\Phi$ is on the horizontal axis. During the structure exchange, probability flows continuously from the integrable region to the chaotic region for the 18 th eigenstate (and vice-versa for the 19th). 


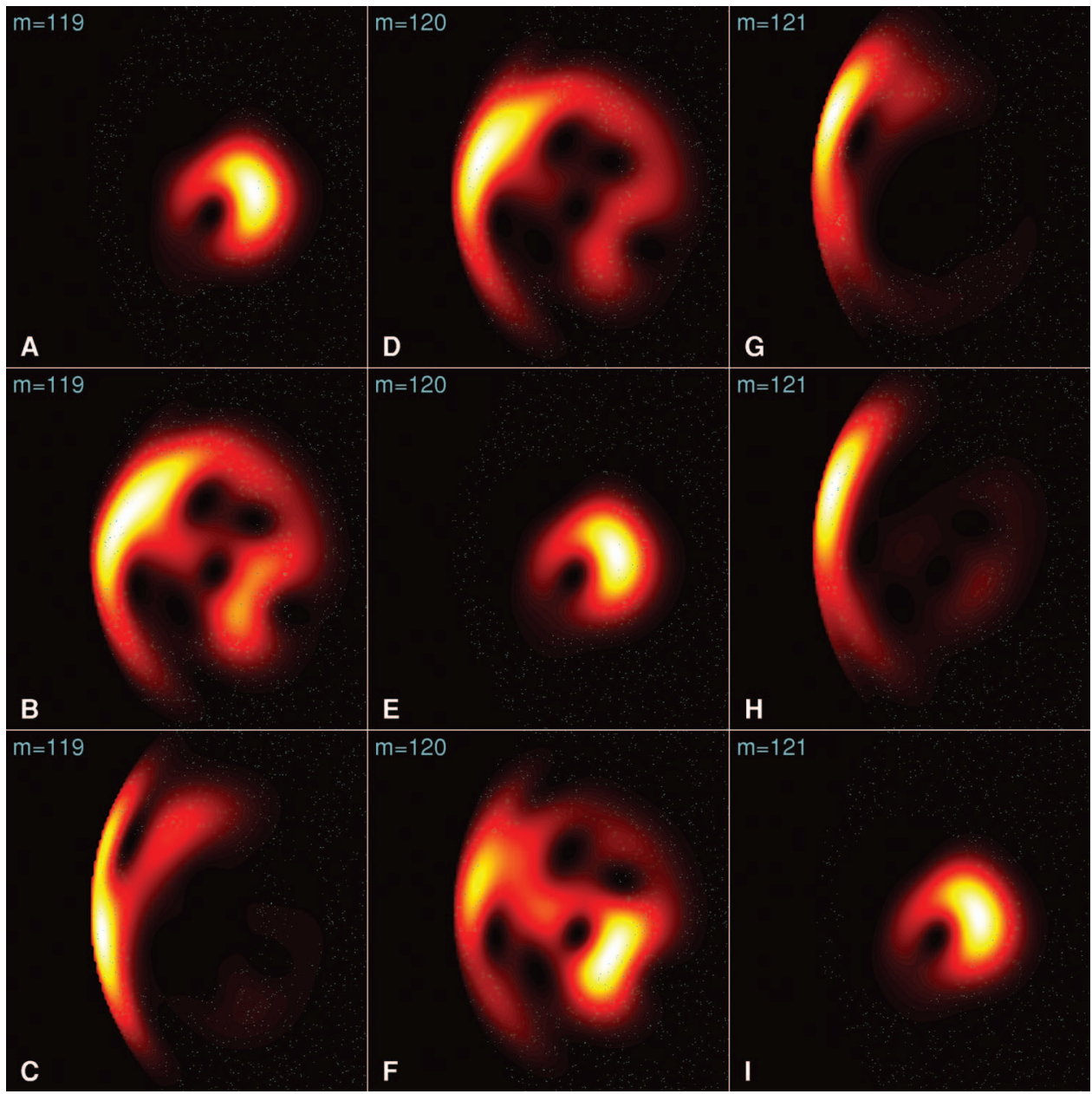

FIG. 10. (Color) Mixing of Husimi structures through the avoidedcrossing cluster of Fig. 5. The left, center, and right columns show the Husimi distributions of the 119th, 120th, and 121st levels, respectively. The axes are as in Fig. 9. as mixtures of the two initial distributions. Additionally, the regular and chaotic portions of the structure are connected. As $\alpha$ is varied, Husimi probability flows continuously between integrable and chaotic regions. The bottom panels, depicting snapshots from long after the encounter, show that the two levels have completely exchanged their structure through the avoided crossing, leaving the aggregate Husimi structure unchanged. This provides an example of a smooth exchange of character in a sharp avoided crossing, which has also been observed in other quantum chaotic systems. ${ }^{38,57}$ The avoided crossings that we have observed between chaotic and regular states and between two chaotic states have all been sharp ones.

\section{Broad avoided crossings}

As described previously, broad avoided crossings in the energy-level curves typically occur in nearly isolated clusters in which the initial slopes of the curves are not fully exchanged after the sequence of avoided crossings. This leads to a mixing of Husimi structures rather than a complete exchange, ${ }^{38}$ which we have verified is the case for the Hamiltonian (5). Figure 10 shows an example of such a mixing in the cluster of avoided crossings from the 119th through the 121st energy levels from Fig. 5. The localization of the initial Husimi structure of the 119th level indicates that this eigenstate is regular. The 120th level is localized near an integrable region, although there is a significant $\mathrm{Hu}-$ simi density in the chaotic sea, and the 121 st level displays a strong chaotic localization with a slight density near the integrable region. The 119th and 120th levels leave the avoided-crossing cluster with Husimi structures that appear as mixtures between the initial Husimi structures of the 120 th and 121 st levels. The 121st state, however, leaves with a Husimi structure nearly identical to the initial structure of the 119th state. These observations are consistent with the slope exchanges in Fig. 5. This mixing causes the Husimi distributions of the 119th and 120th levels to delocalize after the avoided-crossing cluster. Thus, in contrast to sharp avoided crossings, broad avoided-crossing clusters play a significant role in modifying the aggregate Husimi structure as the coupling strength $\alpha$ increases. In particular, such clusters appear to mix the Husimi structure of individual eigenstates.

\section{Localization and $\alpha$-dependence of avoided crossings}

We have observed that sharp avoided crossings involve an interaction either between two chaotic levels or between a level localized primarily in an integrable region (with only slight localization in the chaotic region) and one localized 
primarily in a chaotic region. However, broad avoided crossings such as those in Fig. 5 tend to involve interactions between levels with significant localization in both integrable and chaotic regions.

We have also observed that broad avoided crossings become more prevalent as $\alpha$ increases [see, for example, Fig. 4(a)]. Because clusters of broad avoided crossings lead to mixing of the Husimi structures of the participating levels, one expects that mixing among eigenstates should also become more prominent for larger values of $\alpha$. Additionally, the $\alpha$-step size required to resolve avoided crossings (i.e., to confirm that they are avoided crossings rather than actual level crossings) appears to be correlated with the initial localization of the Husimi distributions of the participating energy levels. Excluding avoided crossings between two chaotic states, the maximum step size that is sufficient for resolution seems to increase as the localization of the participating levels in similar regions of the classical SOS increases. Because adjacent eigenstates with energy-level curves that are nearly parallel over some range of $\alpha$ can be interpreted as an extremely long-range avoided crossing (with a very large step size sufficient for resolution), they provide a limiting case of the above situation. Such examples arise when the participating eigenstates are localized to almost the same extent in the same integrable region.

We have observed a related phenomenon in the rate at which the doubly degenerate eigenvalues at $\alpha=0$ split (see Fig. 2). For example, when $\alpha$ lies strictly between 0 and the first sharp avoided crossing in Fig. 2, the 17th and 18th levels are regular (with Husimi structure similar to panel A of Fig. 9), whereas the 19th and 20th levels are chaotic (with Husimi structure similar to panel D of Fig. 9). The eigenvalues of the two chaotic states clearly diverge from each other faster than those of the two regular states. This observation is reminiscent of that discussed above for avoided crossings except that the eigenenergies are degenerate at some value of $\alpha$, rather than nearly degenerate. Indeed, suppose that one is starting from the point of closest approach of an avoided crossing. As $\alpha$ increases, a broad avoided crossing between two states initially localized primarily in integrable regions exhibits a much smaller rate of divergence from neardegeneracy than a sharp avoided crossing between two chaotic states. We have also observed that degenerate eigenstates always split into either two chaotic states or two regular ones and that degenerate chaotic states always seem to split at a faster rate than degenerate regular ones.

\section{Signatures of chaos}

Because ergodicity and exponentially fast divergence of phase-space trajectories can be used to characterize classical chaotic systems, it has been suggested that delocalization in the Husimi distributions of a quantum system is a possible signature of chaos in its classical counterpart. ${ }^{58}$ This has been quantified and studied in numerical investigations ${ }^{38,59}$ and is also germane to the system investigated here, as the fraction of phase space with chaotic dynamics in the classical system (1) increases with $L .{ }^{1}$ Consequently, the delocalization and mixing of Husimi structures, which both become more prominent as the prevalence of broad avoided crossings increases with $L$, seem to be signatures of the chaos in the corresponding classical system.

\section{CONCLUSIONS}

In this paper, we investigated a model system with mixed regular and chaotic dynamics that consists of the quantization of a one-dimensional free particle on a ring coupled to a one-dimensional harmonic oscillator. By examining the eigenenergies as a function of the system parameters (the coupling strength $\alpha$ and the relative sizes of the interaction and noninteraction regions) and computing $\mathrm{Hu}-$ simi distributions, we studied the quantum signatures of the mixed dynamics. We identified key integrable and chaotic structures of Husimi distributions by comparing them with the corresponding classical surfaces of section. For example, we examined sharp avoided crossings between states localized in chaotic regions and those localized in integrable ones and demonstrated numerically the concomitant complete exchange of their Husimi structures. ${ }^{34,38,57}$ We also showed that an avoided crossing between two mixed states tends to be broader than that between a predominately regular state and a predominately chaotic one. Furthermore, we found that the $\alpha$-step size required to resolve an avoided crossing is correlated with the extent to which the participating states are localized in the chaotic and integrable portions of phase space.

As the size of the noninteraction region increases, the avoided crossings broaden and their density increases. This, in turn, leads to an increase in the number of avoidedcrossing clusters, in which the participating energy-level curves do not fully exchange slopes (as a function of $\alpha$ ). We showed numerically that such avoided-crossing clusters mix the Husimi structures between participating states rather than exchange them fully as in the sharp avoided crossings. Such mixing tends to promote delocalization in the eigenstates as the coupling strength is increased. This causes a nontrivial modification in the aggregate Husimi structure as the coupling strength is varied, in contrast to the preservation of the aggregate structure that is characteristic of sharp avoided crossings. Consequently, as the length of the noninteraction region increases, one observes an increasing amount of mixing in the aggregate Husimi structure as a function of the coupling strength. This is a signature of the dynamics of the corresponding classical system, for which the chaotic portion of phase space increases with the size of the noninteraction region. Thus, the appearance of broad avoided crossings, eigenstate delocalization, and the mixing of Husimi structures seem to be signatures of chaos in the quantum system. Our numerical computations also suggest that the sharpness of avoided crossings is positively correlated with the extent to which the participating Husimi structures are localized in different regions of phase space. Therefore, the dynamics of avoided crossings in quantum systems seems to be strongly related to the chaotic dynamics of their classical counterparts. 


\section{ACKNOWLEDGMENTS}

We thank Stephan De Bièvre, Jens Marklof, Jerry Marsden, Alex McCauley, Kevin Mitchell, Paul Parris, Alex Silvius, and Turgay Uzer for useful discussions concerning this research. We also thank Alex Barnett, Mark Dykman, Joachim Stolze, and two anonymous referees for providing numerous critical comments and suggestions regarding this manuscript. T.M. acknowledges support from Caltech's Summer Undergraduate Research Fellowship (SURF) Program and the Richter Memorial Funds. M.A.P. acknowledges support from the Gordon and Betty Moore Foundation through Caltech's Center for the Physics of Information (where he held a postdoctoral position during this research).

\section{APPENDIX A: THE HAMILTONIAN MATRIX}

Let $\mathcal{H}_{1}=\mathcal{H}_{\text {part }}$ be the Hilbert space for a free particle traversing a ring of length $2+L$ and $\mathcal{H}_{2}=\mathcal{H}_{\text {osc }}$ be the Hilbert space for a harmonic oscillator. Define $|n\rangle_{1}=\left|\psi_{n}^{\text {part }}\right\rangle$ and $E_{n}^{1}$, respectively, to be the $n$th eigenstate and corresponding $n$th eigenenergy for the particle. We calculate the coordinatespace projections $\left\{\psi_{n}^{\text {part }}(q), \psi_{-n}^{\text {part }}(q)\right\}_{n=1}^{\infty}$ of $\left\{|n\rangle_{1},|-n\rangle_{1}\right\}_{n=1}^{\infty}$ and their eigenenergies from the Schrödinger equation with periodic boundary conditions:

$$
\begin{aligned}
& \frac{\partial^{2}}{\partial q^{2}} \psi_{n}^{\text {part }}(q)=-E_{n}^{1} \psi_{n}^{\text {part }}(q), \\
& \psi_{n}^{\text {part }}(q+l(2+L))=\psi_{n}^{\text {part }}(q), \quad l \in \mathbb{Z} .
\end{aligned}
$$

The (normalized) solutions are

$$
\begin{aligned}
& \psi_{n}^{\text {part }}(q)=\frac{1}{\sqrt{2+L}} \exp \left\{\left(\frac{2 \pi n i}{2+L}\right) q\right\}, \\
& E_{n}^{1}=\frac{4 \pi^{2} n^{2}}{(2+L)^{2}}, \quad n \in \mathbb{Z}-\{0\} .
\end{aligned}
$$

The ground-state energy of the particle is $E_{ \pm 1}^{1}=4 \pi^{2}$ / $\left[(2+L)^{2}\right]$.

Define $|k\rangle_{2}=\left|\psi_{k}^{\text {osc }}\right\rangle$ and $E_{k}^{2}$, respectively, to be the $k$ th eigenstate and corresponding $k$ th eigenenergy of the oscillator. Here, $E_{k}^{2}=k+1 / 2$, so that $E_{0}^{2}=1 / 2$ is the ground-state energy. ${ }^{31}$ Using the operator definitions (4), we write

$$
a^{\dagger}=\frac{1}{\sqrt{2}}(\Phi-i \Pi), \quad a=\frac{1}{\sqrt{2}}(\Phi+i \Pi),
$$

which are, respectively, the creation and annihilation operators for the harmonic oscillator. ${ }^{31,60}$ The Hamiltonian (5) becomes

$$
H=\left(a^{\dagger} a+\frac{1}{2}\right)+\frac{p^{2}}{2}-\frac{\alpha}{\sqrt{2}}\left(a^{\dagger}+a\right) \chi(q) .
$$

The matrix representation of Eq. (A2) in the uncoupled basis $|n\rangle_{1} \otimes|k\rangle_{2}$ \{with integer indices $n \in(-\infty,-1] \cup[1, \infty)$ and $k$ $\in[0, \infty)\}$ is

$$
\mathbf{H}=\mathbf{E}_{\mathbf{1}} \otimes \mathbb{I}+\mathbb{I} \otimes \mathbf{E}_{\mathbf{2}}-\alpha \mathbf{W}_{\mathbf{1}} \otimes \mathbf{W}_{\mathbf{2}},
$$

where $I$ is the identity matrix and

$$
\begin{aligned}
& \left(\mathbf{E}_{1}\right)_{n n^{\prime}}=\left\langle n\left|\frac{p^{2}}{2}\right| n^{\prime}\right\rangle_{1}, \\
& \left(\mathbf{E}_{2}\right)_{k k^{\prime}}=\left\langle k\left|\left(a^{\dagger} a+\frac{1}{2}\right)\right| k^{\prime}\right\rangle_{2}, \\
& \left(\mathbf{W}_{\mathbf{1}}\right)_{n n^{\prime}}=\left\langle n|\chi(q)| n^{\prime}\right\rangle_{1}, \\
& \left(\mathbf{W}_{\mathbf{2}}\right)_{k k^{\prime}}=\left\langle k\left|\frac{1}{\sqrt{2}}\left(a^{\dagger}+a\right)\right| k^{\prime}\right\rangle_{2} .
\end{aligned}
$$

By the definition of the uncoupled basis,

$$
\left(\mathbf{E}_{\mathbf{1}}\right)_{n n^{\prime}}=\frac{4 \pi^{2} n^{2}}{(2+L)^{2}} \delta_{n n^{\prime}}, \quad\left(\mathbf{E}_{2}\right)_{k k^{\prime}}=\left(k+\frac{1}{2}\right) \delta_{k k^{\prime}} .
$$

The coordinate-space projections for the free-particle eigenstates yield

$$
\begin{aligned}
\left(\mathbf{W}_{1}\right)_{n n^{\prime}} & =\int_{0}^{2+L} \psi_{n}^{\text {part }}(q)^{*} \psi_{n^{\prime}}^{\text {part }}(q) \chi(q) d q \\
& =\int_{0}^{2} \psi_{n}^{\text {part }}(q)^{*} \psi_{n^{\prime}}^{\text {part }}(q) d q .
\end{aligned}
$$

Hence, with Eq. (A1), we obtain

$$
\left(\mathbf{W}_{1}\right)_{n n^{\prime}}= \begin{cases}\frac{1}{2 \pi\left(n-n^{\prime}\right)}\left(-i+i e^{4 \pi i\left(n-n^{\prime}\right) / 2+L}\right) & \text { if } n \neq n^{\prime}, \\ \frac{2}{2+L} & \text { if } n=n^{\prime} .\end{cases}
$$

Finally, the creation/annihilation operator identities

$$
a^{\dagger}|k\rangle_{2}=\sqrt{k+1}|k+1\rangle_{2}, \quad a|k\rangle_{2}=\sqrt{k}|k-1\rangle_{2}
$$

give

$$
\left(\mathbf{W}_{2}\right)_{k k^{\prime}}=\frac{1}{\sqrt{2}}\left(\sqrt{k^{\prime}+1} \delta_{k, k^{\prime}+1}+\sqrt{k^{\prime}} \delta_{k, k^{\prime}-1}\right) .
$$

\section{APPENDIX B: THE HUSIMI DISTRIBUTION}

The Husimi distribution $H_{\psi}(\bar{p}, \bar{q}, \bar{\Phi}, \bar{\Pi})$ of a state $|\psi\rangle$ of the two-dimensional quantum-mechanical system (5) is

$$
H_{\psi}(\bar{p}, \bar{q}, \bar{\Phi}, \bar{\Pi})=N\left|\left\langle\psi_{(\bar{p}, \bar{q}, \bar{\Phi}, \bar{\Pi})} \mid \psi\right\rangle\right|^{2},
$$

where $\left|\psi_{(\bar{p}, \bar{q}, \bar{\Phi}, \bar{\Pi})}\right\rangle$ is a coherent state localized around $(\bar{p}, \bar{q}, \bar{\Phi}, \bar{\Pi})$ and $N$ is a normalization constant. We construct a coherent state for the coupled system as the product

$$
\left|\psi_{(\bar{p}, \bar{q}, \bar{\Phi}, \bar{\Pi})}\right\rangle=\left|\psi_{(\bar{p}, \bar{q})}\right\rangle_{1} \otimes\left|\psi_{(\bar{\Phi}, \bar{\Pi})}\right\rangle_{2},
$$

where $\left|\psi_{(\bar{p}, \bar{q})}\right\rangle_{1}$ is the coherent state for the free particle and $\left|\psi_{(\bar{\Phi}, \bar{\Pi})}\right\rangle_{2}$ is the coherent state for the uncoupled harmonic oscillator. The latter is given by ${ }^{31}$ 


$$
\left|\psi_{(\bar{\Phi}, \bar{\Pi})}\right\rangle_{2}=e^{-\left(\bar{\Phi}^{2}+\bar{\Pi}^{2}\right) / 2} \sum_{k=0}^{\infty} \frac{(\bar{\Phi}+i \bar{\Pi})^{k}}{\sqrt{k !}}|k\rangle_{2} .
$$

Because $p \in \mathbb{R}$ and $q$ is $(2+L)$-periodic in the uncoupled particle system, the phase space is cylindrical. Using the procedure of Ref. 48 to define $\left|\psi_{(\bar{p}, \bar{q}, \bar{\Phi}, \bar{\Pi})}\right\rangle$ for this topology, we require that the coherent state $\left|\psi_{(\bar{p}, \bar{q})}\right\rangle_{1}$ satisfies

$$
\left\langle q \mid \psi_{(\bar{p}, \bar{q})}\right\rangle_{1}=\left\langle q+l(2+L) \mid \psi_{(\bar{p}, \bar{q})}\right\rangle_{1}, \quad l \in \mathbb{Z} .
$$

One can construct the coherent states $\left|\psi_{(\bar{p}, \bar{q},}\right\rangle_{1}$ from Euclidean coherent states $\left|\widetilde{\psi}_{(\bar{p}, \bar{q})}\right\rangle_{1}$ (which are Gaussian wavefunctions) by wrapping them around the cylinder and summing overlapping portions. This yields

$$
\left\langle q \mid \psi_{(\bar{p}, \bar{q})}\right\rangle_{1}=C^{1 / 2} \sum_{l=-\infty}^{\infty}\left\langle q+l(2+L) \mid \tilde{\psi}_{(\bar{p}, \bar{q})}\right\rangle_{1},
$$

which satisfies Eq. (B4) and converges because $\langle q+l(2$ $+L)\left|\tilde{\psi}_{(p, q)}\right\rangle_{1}$ is Gaussian. In Eq. (B5), the quantity $C$ is a normalization constant to be determined by the condition $\left\langle\psi_{(\bar{p}, \bar{q})} \mid \psi_{(\bar{p}, \bar{q})}\right\rangle_{1}=1$.

The projection of $\left|\psi_{(\bar{p}, \bar{q})}\right\rangle_{1}$ onto the uncoupled particle basis $\left\{|n\rangle_{1},|-n\rangle_{1}\right\}_{n=1}^{\infty}$ is

$$
\left\langle n \mid \psi_{(\bar{p}, \bar{q})}\right\rangle_{1}=\int_{0}^{2+L}\langle n \mid q\rangle_{1}\left\langle q \mid \psi_{(\bar{p}, \bar{q})}\right\rangle_{1} d q=C^{1 / 2} \sum_{l=-\infty}^{\infty} \int_{0}^{2+L}\langle n \mid q+l(2+L)\rangle_{1}\left\langle q+l(2+L) \mid \widetilde{\psi}_{(\bar{p}, \bar{q})}\right\rangle_{1} d q
$$

Using Eq. (A1) and the $(2+L)$-periodicity of $\psi_{n}^{\text {part }}(q)$ $=\langle q \mid n\rangle_{1}$, we obtain

$$
\left\langle n \mid \psi_{(\bar{p}, \bar{q})}\right\rangle_{1}=\int_{-\infty}^{\infty}\langle n \mid q\rangle_{1}\left\langle q \mid \widetilde{\psi}_{(\bar{p}, \bar{q})}\right\rangle_{1} d q
$$

The coordinate-space projection of the Euclidean coherent state is

$\left\langle q \mid \widetilde{\psi}_{(\bar{p}, \bar{q})}\right\rangle_{1}=\left(\frac{1}{\pi}\right)^{1 / 4} \exp \left[-\frac{1}{2}(q-\bar{q})^{2}+i \bar{p}\left(q-\frac{\bar{q}}{2}\right)\right]$.

Using the expression for $\langle q \mid n\rangle_{1}$ from Eq. (A1), we evaluate Eq. (B6) and obtain

$\left\langle n \mid \psi_{(\bar{p}, \bar{q})}\right\rangle_{1}=C^{1 / 2}\left(\frac{1}{2}\right)^{1 / 4} \exp \left[-\frac{1}{2}(n-\bar{p})^{2}-i \bar{q}\left(n-\frac{\bar{p}}{2}\right)\right]$.

With the normalization condition $\left\langle\psi_{(\bar{p}, \bar{q})} \mid \psi_{(\bar{p}, \bar{q})}\right\rangle_{1}=1$, we determine from (B8) that

$$
C=\sqrt{\pi} \sum_{n \neq 0} e^{-(n-\bar{p})^{2}} .
$$

Thus, if a state $|\psi\rangle$ is expressed in the uncoupled basis as $|\psi\rangle=\sum_{n \neq 0} \sum_{k=0}^{\infty} a_{n k}|n\rangle_{1} \otimes|k\rangle_{2}$, we obtain from Eqs. (B1), (B3), and (B8) that

$$
\begin{aligned}
H_{\psi}(\bar{p}, \bar{q}, \bar{\Phi}, \bar{\Pi})= & \frac{C(\bar{p})}{\sqrt{2}} \mid \sum_{n \neq 0} \sum_{k=0}^{\infty} a_{n k}^{*} \frac{(\bar{\Phi}+i \bar{\Pi})^{k}}{\sqrt{k !}} \\
& \times \exp \left[-\frac{1}{2}\left[\bar{\Phi}^{2}+\bar{\Pi}^{2}+(n-\bar{p})^{2}\right]\right. \\
& \left.-i \bar{q}\left(n-\frac{\bar{p}}{2}\right)\right]\left.\right|^{2} .
\end{aligned}
$$

In practice, we truncate the sum in Eq. (B10) in order to compute the Husimi distribution for eigenstates calculated using a truncated Hamiltonian matrix for Eq. (5). To compare Husimi distributions with classical Poincaré surfaces of section, we take $\bar{p}=\left(2 E-\bar{\Phi}^{2}-\bar{\Pi}^{2}+2 \alpha \bar{\Phi}\right)^{1 / 2}$ (the eigenstate has energy $E$ ) and an appropriate value of $\bar{q}$ (with, for example, $\bar{q}=1$ for a section in the center of the interaction region). The value for $\bar{p}$ arises as a slice along the classical energy shell, and the value for $\bar{q}$ corresponds to the choice of Poincare SOS for the classical system. ${ }^{1}$

${ }^{1}$ S. De Bièvre, P. E. Parris, and A. A. Silvius, Physica D 208, 96 (2005).

${ }^{2}$ M. C. Gutzwiller, Chaos in Classical and Quantum Mechanics (SpringerVerlag, New York, 1990).

${ }^{3}$ F. Haake, Quantum Signatures of Chaos, 2nd ed. (Springer-Verlag, Berlin, 2001).

${ }^{4}$ C. M. Marcus, A. J. Rimberg, R. M. Westervelt, P. F. Hopkins, and A. C. Gossard, Phys. Rev. Lett. 69, 506 (1992).

${ }^{5}$ L. E. Reichl, The Transition to Chaos: Conservative Classical Systems and Quantum Manifestations, 2nd ed. (Springer-Verlag, New York, 2004).

${ }^{6}$ C. Zhang, J. Liu, M. G. Raizen, and Q. Niu, Phys. Rev. Lett. 93, 074101 (2004).

${ }^{7}$ A. Kudrolli, V. Kidambi, and S. Sridhar, Phys. Rev. Lett. 75, 822 (1995).

${ }^{8}$ H. J. Stöckmann and J. Stein, Phys. Rev. Lett. 64, 2215 (1990).

${ }^{9}$ M. F. Anderson, A. Kaplan, T. Grünzweig, and N. Davidson, Phys. Rev. Lett. 97, 104102 (2006).

${ }^{10}$ N. Friedman, A. Kaplan, D. Carasso, and N. Davidson, Phys. Rev. Lett. 86, 1518 (2001).

${ }^{11}$ A. H. Barnett and T. Betcke, Chaos 17, 043125 (2007).

${ }^{12}$ M. V. Berry, Eur. J. Phys. 2, 91 (1981).

${ }^{13}$ L. A. Bunimovich, Chaos 11, 802 (2001).

${ }^{14}$ M. A. Porter and S. Lansel, Not. Am. Math. Soc. 53, 334 (2006).

${ }^{15}$ N. Saitô, H. Hirooka, J. Ford, F. Vivaldi, and G. H. Walker, Physica D 5, 273 (1982).

${ }^{16}$ E. Fermi, Phys. Rev. 75, 1169 (1949).

${ }^{17}$ A. J. Lichtenberg and M. A. Lieberman, Regular and Chaotic Dynamics, 2nd ed. (Springer-Verlag, New York, 1992).

${ }^{18}$ P. Seba, Phys. Rev. A 41, 2306 (1990).

${ }^{19}$ F. Haake, M. Kuś, and R. Scharf, Z. Phys. B: Condens. Matter 65, 381 (1987).

${ }^{20}$ A. Iomin, S. Fishman, and G. M. Zaslavsky, Phys. Rev. E 65, 036215 (2002).

${ }^{21}$ F. L. Moore, J. C. Robinson, and C. F. Bharucha, Phys. Rev. Lett. 75, 4598 (1995).

${ }^{22}$ A. O. Caldeira and A. J. Leggett, Ann. Phys. (Paris) 149, 374 (1983).

${ }^{23}$ R. P. Feynman, Phys. Rev. 97, 660 (1955). 
${ }^{24}$ H. Frölich, Adv. Phys. 3, 325 (1954).

${ }^{25}$ T. Holstein, Ann. Phys. (Paris) 8, 325 (1959).

${ }^{26}$ T. Holstein, Ann. Phys. (Paris) 8, 343 (1959).

${ }^{27}$ V. M. Kenkre and D. K. Campbell, Phys. Rev. B 34, 4959 (1986).

${ }^{28}$ V. M. Kenkre and L. Giuggioli, Chem. Phys. 296, 135 (2004).

${ }^{29}$ L. Müller, J. Stolze, H. Leschke, and P. Nagel, Phys. Rev. A 44, 1022 (1991).

${ }^{30}$ A. A. Silvius, P. E. Parris, and S. De Bièvre, Phys. Rev. B 73, 014304 (2006).

${ }^{31}$ C. Cohen-Tannoudji, B. Diu, and F. Laloë, Quantum Mechanics (Wiley, New York, 1977), Vol. 1

${ }^{32}$ W.-M. Zhang, D. H. Feng, J.-M. Yuan, and S.-J. Wang, Phys. Rev. A 40, 438 (1989)

${ }^{33}$ W.-M. Zhang, C. C. Martens, D. H. Feng, and J.-M. Yuan, Phys. Rev. Lett. 61, 2167 (1988)

${ }^{34}$ J. von Neumann and E. Wigner, in Symmetry in the Solid State, edited by R. S. Knox and A. Gold (W. A. Benjamin, New York, 1964) [translated from Phys. Z. 30, 467 (1929)].

${ }^{35}$ R. González-Férez and J. S. Dehesa, Eur. Phys. J. D 32, 39 (2005).

${ }^{36}$ D. W. Hone, R. Ketzmerick, and W. Kohn, Phys. Rev. A 56, 4045 (1997).

${ }^{37}$ B. Ramachandran and K. G. Kay, J. Chem. Phys. 99, 3659 (1993).

${ }^{38}$ T. Timberlake and L. E. Reichl, Phys. Rev. A 59, 2886 (1999).

${ }^{39}$ J. Wiersig, Phys. Rev. Lett. 97, 253901 (2006).

${ }^{40}$ We use the term "isolated cluster" to designate a group of nearby avoided crossings whose participating energy levels are nearly unaffected by other levels (outside the group) in their "incoming" (as a function of $\alpha$ ) and outgoing regions.
${ }^{41}$ D. W. Noid, M. L. Koszykowski, M. Tabor, and R. A. Marcus, J. Chem. Phys. 72, 6169 (1980).

${ }^{42}$ T. Uzer, D. W. Noid, and R. A. Marcus, J. Chem. Phys. 79, 4412 (1983)

${ }^{43}$ H.-J. Stöckmann, Quantum Chaos: An Introduction (Cambridge University Press, Cambridge, UK, 1999).

${ }^{44}$ M. V. Berry and M. Robnik, J. Phys. A 17, 2413 (1984).

${ }^{45}$ J. Malovrh and T. Prosen, J. Phys. A 35, 2483 (2002).

${ }^{46}$ T. Prosen and M. Robnik, J. Phys. A 26, 2371 (1993).

${ }^{47}$ Y. Weissman and J. Jortner, J. Chem. Phys. 77, 1486 (1982).

${ }^{48}$ A. Spina and R. T. Skodje, Comput. Phys. Commun. 63, 279 (1991).

${ }^{49}$ A. Bäcker, S. Fürstberger, and R. Schubert, Phys. Rev. E 70, 036204 (2004)

${ }^{50}$ S. Wiggins, Introduction to Applied Nonlinear Dynamical Systems and Chaos, 2nd ed. (Springer-Verlag, New York, 2003).

${ }^{51}$ S.-J. Chang and G. Perez, Chin. J. Phys. (Taipei) 30, 479 (1992).

${ }^{52}$ S.-J. Chang and M. Stuller, Chin. J. Phys. (Taipei) 35, 469 (1997).

${ }^{53}$ L. Perotti, Phys. Rev. E 70, 066218 (2004).

${ }^{54}$ A. Bäcker, R. Schubert, and P. Stifter, Phys. Rev. E 57, 5425 (1998).

${ }^{55}$ I. C. Percival, J. Phys. B 6, L229 (1973).

${ }^{56}$ J. Marklof and S. O'Keefe, Nonlinearity 18, 277 (2005).

${ }^{57}$ B. P. Holder and L. E. Reichl, Phys. Rev. A 72, 043408 (2005).

${ }^{58}$ A. Sugita, J. Phys. A 36, 9081 (2003).

${ }^{59}$ A. Sugita and H. Aiba, Phys. Rev. E 65, 036205 (2002).

${ }^{60} \mathrm{R}$. Shankar, Principles of Quantum Mechanics, 2nd ed. (Plenum, New York, 1994). 